\title{
Caractéristiques du feutrage algal des récifs coralliens de Polynésie française soumis à différentes intensités de bioérosion
}

\author{
Sylvain LE BRIS, Thérèse LE CAMPION-ALSUMARD, Jean-Claude ROMANO
}

Centre d'océanologie de Marseille, université de la Méditerranée UMR CNRS 6540, Station marine d'Endoume, rue de la Batterie-des-Lions, 13007 Marseille, France

(Reçu le 03/10/97, révisé le 06/02/98, accepté le10/03/98)

\begin{abstract}
Characteristics of epilithic and endolithic algal turf exposed to different levels of bioerosion in French Polynesian coral reefs. This study deals with the colonisation of experimental Porites blocks by epilithic and endolithic algal community in two atoll lagoons and two high-island lagoons in French Polynesia, exposed to different environmental conditions during a 5-year period. A qualitative analysis of the community (cyanobacteria and micro-algae) was carried out, and the relationship between the epilithic and endolithic strata observed. IIPLC analysis of chlorophyll pigments was used to quantitatively determine the plant biomass, which was then compared to that of the algal turf of neighbouring reefs. The results show that these blocks are colonised according to two factors: the reef structure (atoll or high-island) and the degree of human disturbance. The epilithic and endolithic population dynamics differ.

The density of the epilithic algal community and the species diversity increase with the degree of eutrophication and the amount of borers (sea-urchins, parrotfish), while the proportion of endolithic species remains low (34\%-59\%). This is especially marked in the high-island lagoons, where leaching has caused enhanced nutrient levels. An extreme case is the station at Faaa, a highly polluted harbour, where increased sea-urchin levels ( 44 ind. $\mathrm{m}^{-2}$ ) meant that the blocks disappeared completely in less than five years due to bioerosion. In the oligotrophic waters of the atoll lagoons, the losses of $\mathrm{CaCO}_{3}$ by biocrosion do not excecd $35 \%$ per block, and the algal community is made up mainly of cndolithic specics ( $66 \%-85 \%$ ). The algal biomass is lowest in the Takapoto atolls $\left(10-11 \mu \mathrm{g} \mathrm{cm}^{-2}\right.$ ) and maximum in Tikehau atoll $\left(23\right.$ and $56 \mu \mathrm{g} \mathrm{cm}^{-2}$ ). The first microborers to settle in the ecological succession of the community are the cyanobacteria. These are gradually replaced by Ostreobium (Chlorophyceae borers), which become dominant in the blocks exposed to the lowest bioerosion rate. The richness and the density of the algal turf reflect the convergence of many ecological factors: it seems that the covering of epilithic algae or the richness of endolithic flora could be used as a global indicator of the quality and the health of a reef. (c) Elsevier, Paris
\end{abstract}

coral reef / epilithic and endolothic algal community / biomass / bioerosion / eutrophication

Résumé - Cette étude porte sur la colonisation par du feutrage algal épilithe et endolithe de blocs expérimentaux de Porites, mis en place dans deux atolls et deux îles hautes de Polynésie française et soumis à des contraintes environnementales différentes pendant cinq ans. La composition qualitative des peuplements (cyanobactéries et microalgues) et les relations entre «strate » épilithe et «strate » endolithe ont été observées. La quantification de la biomasse végétale a été effectuée par la mesure des pigments chlorophylliens analysés en HPLC. Des comparaisons ont été établies avec le feutrage algal des substrats récifaux avoisinants. Les résultats font apparaître des différences dans l'évolution de la coloni- 
sation de ces blocs selon deux facteurs: la structure récifale (atolls ou îles hautes) et le degré d'anthropisation des milieux. Par ailleurs, la dynamique de la colonisation est différente pour les populations épilithes et endolithes.

La densité du feutrage algal épilithe et la diversité spécifique augmentent avec le degré d'eutrophisation des eaux et la quantité des organismes brouteurs (oursins, Scaridés), alors que la contribution des espèces endolithes dans ce feutrage reste faible (34 et $59 \%$ ). C'est le cas notamment dans les stations des îles hautes, enrichies en nutriments par le lessivage des sols. L'exemple extrême est représenté par la station de Faaa (ville de Papeete) où la forte pollution urbaine et le nombre élevé d'oursins bioérodeurs ( $>44$ ind. $\mathrm{m}^{2}$ ) ont entraîné la disparition des blocs en moins de cinq ans. Dans les atolls, les eaux sont oligotrophes, les pertes de $\mathrm{CaCO}_{3}$ par bioérosion ne dépassent pas $35 \%$ par bloc, et le peuplement algal est constitué principalement d'espèces endolithes (66\% à $85 \%$ de l'ensemble du peuplement). Sur les blocs de l'atoll de Takapow, la biomasse du feutrage algal épilithe et endolithe est la plus faible et n'atteint que 10 à $11 \mu \mathrm{g} \mathrm{chl} a \mathrm{~cm}^{-2}$, alors qu'elle est maximale (23 et $56 \mu \mathrm{g} \mathrm{chl} a \mathrm{~cm}^{-2}$ ) aux deux stations de l'atoll de Tikehau. Dans la succession écologique, les premiers microperforants qui s'installent sont les cyanobactéries; celles-ci sont peu à peu remplacées par l'Ostreobium (Chlorophycée perforante) qui devient dominant dans les blocs soumis à la plus faible bioérosion. La richesse et la densité du feutrage algal reflétant l'intégration de nombreux facteurs écologiques, il apparaît que la couverture en épilithes ou la richesse en flore endolithe pourraient servir d'indicateur global de la qualité et de la santé d'un récif. Đ Elsevier, Paris

\section{récif corallien / feutrage algal épilithe et endolithe / biomasse / bioérosion / eutrophisation}

\section{INTRODUCTION}

Dans les récifs coralliens, deux phénomènes antagonistes interviennent dans le cycle des carbonates : la fixation du $\mathrm{CaCO}_{3}$ pour la bioconstruction (coraux, algues calcaires) et sa libération dans l'eau par action mécanique, chimique et biologique. La bioérosion fait l'objet de ce travail, et plus spécifiquement son influence sur la composition et la biomasse du feutrage algal.

La bioérosion des substrats carbonatés dépend directement ou indirectement de l'importance du feutrage algal épilithe et endolithe qui recouvre ces substrats. En effet, dans le milieu marin, tout type de substrat vierge immergé est rapidement recouvert d'un feutrage algal épilithe constitué dc cyanobactérics ct d'algues de très petite taille $(<5 \mathrm{~mm})$, et d'une couche endolithe présente uniquement à l'intérieur des substrats carbonatés [19]. Il en est de même dans les récifs coralliens. Le feutrage algal épilithe et endolithe est à la base de la nourriture de nombreux organismes brouteurs, comme les oursins et les Scaridés, plutôt attirés par les substrats coralliens recouverts par les cyanobactéries et les algues molles épilithes $[1,23]$. Les algues se développant à l'intérieur des substrats carbonatés, dissolvent une partie du $\mathrm{CaCO}_{3}$ [20]. Les organismes brouteurs se nourrissant de cette strate endolithe arrachent des fragments de substrat et accentuent cette bioérosion. A cette bioérosion s'ajoute une érosion mécanique favorisée par le réseau d'endolithes qui augmente la porosité et la fragilité du calcaire. Ces endolithes, présents à la fois dans les coraux morts et vivants, jouent donc un rôle significatif dans la bioérosion et dans la production primaire, mais ils sont peu étudiés.

La densité du feutrage algal épilithe est le reflet du broutage. Les herbivores en contrôlent la distribution, l'abondance et la hauteur $[3,10]$. En outre, le broutage augmente la production en diminuant l'auto-ombrage, en sélectionnant les espèces les plus résistantes et en augmentant la disponibilité en nutriments au travers des déchets organiques [15]. Les rares études de la composition du feutrage algal épilithe montrent que les cyanobactérics peuvent représenter jusqu'à $73 \%$ du peuplement [10] dont plusieurs espèces ont la capacité de fixer l'azote. Williams et Carpenter [36] observent que le feutrage algal peut constituer 70 à $80 \%$ de la production primaire totale d'un récif. Scott et Russ [30] précisent que plus ce feutrage est abondant et plus la richesse spécifique est importante. Les variations de la quantité d'organismes brouteurs et l'état de dégradation d'un récif ont des conséquences sur la composition de ce feutrage.

Pour appréhender les importances respectives de la bioconstruction et de la biodestruction dans ces mécanismes complexes, une approche expérimentale, centrée sur la bioérosion, a ćté cntrcprise en Polynésie française sur des blocs de Porites [26]. Dans ce cadre, le présent travail tente d'estimer, après cinq ans de colonisation de ces blocs, les variations qualitatives et quantitatives du peuplement algal endolithe et épilithe, ainsi que leurs interactions avec l'intensité de la bioérosion et les conditions environnementales. 


\section{MATÉRIEL ET MÉTHODES}

\subsection{Localisation des stations}

Sept sites ont été retenus en Polynésie française dans le Pacifique Sud, en raison de leurs structures récifales différentes et d'un gradient de dégradation du milieu. A chaque station est attribué un indice qualitatif de pression anthropique (indice de pollution), établi à partir de facteurs biotiques (concentration en chlorophylle $a$, nombre d'oursins bioérodeurs et de coliformes fécaux) et de facteurs abiotiques (concentration en nitrates, phosphates, silicates, détergents et pesticides). La plage de cet indice va de 1 , pollution la plus faible, à 4 , la plus forte (tableau I). Trois sites se trouvent sur des îles hautes (Tahiti, $17^{\circ} 40^{\prime} \mathrm{S}$ et $149^{\circ} 30^{\prime} \mathrm{W}$ et Moorea, $17^{\circ} 30^{\prime} \mathrm{S}$ et $149^{\circ} 50^{\prime} \mathrm{W}$ ) et quatre dans des lagons d'atoll (Takapoto, $14^{\circ} 30^{\prime} \mathrm{S}$ et $145^{\circ} 20^{\prime} \mathrm{W}$ et Tikehau, $15^{\circ} 00^{\prime} \mathrm{S}$ et $148^{\circ} 10^{\prime}$ W) (figure 1). A Tahiti, un site correspond aux eaux très polluées du port de Papeete (Faaa) et l'autre au récif frangeant d'Atimaono (Atim), un secteur peu urbanisé. A Moorea (Moor), le site choisi correspond au récif frangeant de Tiahura, secteur peu atteint par l'activité humaine. Deux sites ont été retenus dans les lagons des atolls de Takapoto (Tik1 et Tik2) et de Tikehau (Tik1 et Tik2). Pour chaque atoll, un site est proche d'un village
(Tik1 et Tak2) alors que l'autre est dans un secteur de très faible influence anthropique (Tik2 et Tak1).

\subsection{Méthodes d'étude}

\subsubsection{Substrats expérimentaux}

Les substrats expérimentaux sont des blocs parallélépipédiques issus du cœur de grandes colonies de Porites sains. Ils ont été nettoyés de toute trace de matière organique à l'hypochlorite de sodium, rincés soigneusement à l'eau douce puis séchés. Après avoir été pesés et mesurés, ils ont été fixés, dans les sept stations, à l'aide de ciment marin sur des colonies mortes de Porites, à une profondeur de $1 \mathrm{~m}$ environ. En novembre 1995, après cinq ans de colonisation, les blocs ont été retirés et un fragment de substrat naturel où ils étaient fixés a été prélevé. Une partie des blocs et du substrat naturel est lyophilisée pour la quantification des pigments, le reste est conservé dans du formol (3\% dans de l'eau de mer) pour les études taxonomiques.

\subsubsection{Chromatographie des pigments chlorophylliens}

Un à quatre éclats lyophylisés, issus des blocs expérimentaux et des substrats naturels sont soigneusement pesés, mesurés puis broyés dans un mortier en agate et la poudre ainsi obte-

Tableau I. Indice de pollution établis à partir de données de la littérature sur quelques caractéristiques du milieu marin des différentes stations étudiées.

Table I. Pollution index and compiled data on some characteristics of sea water at the study sites.

\begin{tabular}{|c|c|c|c|c|c|c|}
\hline & FAAA & ATIMAONO & MOOREA & TAKAPOTO & TIKEHAU & OCEAN \\
\hline $\begin{array}{l}\text { Nitrate } \\
\left(\text { mmol.L } L^{-1}\right)\end{array}$ & & & $0,50(\mathrm{~d})$ & $0,20(\mathrm{e})$ & 0,08 (f) & $0,06-0,20(\mathrm{~g})$ \\
\hline $\begin{array}{l}\text { Phosphate } \\
\left(\mathrm{mmol} \cdot \mathrm{L}^{-1}\right)\end{array}$ & $0,52(\mathrm{c})$ & & $0,46(d)$ & $0,10(\mathrm{e})$ & $0,16(f)$ & $0,30-0,45(\mathrm{~g})$ \\
\hline $\begin{array}{l}\text { Silicate } \\
\left(\text { mmol.L } L^{-1}\right)\end{array}$ & $7,86(\mathrm{c})$ & & $0,58(d)$ & $0,50(\mathrm{e})$ & 0,83 (f) & $1,80-2,50(\mathrm{~g})$ \\
\hline $\begin{array}{l}\text { Chlorophylle } a \\
\left(\mu \mathrm{g} \cdot \mathrm{L}^{-1}\right)\end{array}$ & $0,31(\mathrm{c})$ & & $0,20(d)$ & $0,13-0,40$ (e) & 0,20 (f) & $0,10-0,20(\mathrm{~g})$ \\
\hline $\begin{array}{l}\text { Oursins (a) } \\
\left(\mathrm{m}^{-2}\right)\end{array}$ & 44 & 2 & 1,5 & 0 & 0 & 0 \\
\hline $\begin{array}{l}\text { Coliformes fécaux (b) } \\
\left(100 \mathrm{~mL}^{-1}\right)\end{array}$ & 10000 & 0 & & & & 0 \\
\hline $\begin{array}{l}\text { Détergents anioniques (b) } \\
\left(\mu \mathrm{g} \cdot \mathrm{kg}^{-1}\right)\end{array}$ & 460 & $10-20$ & & & & 0 \\
\hline $\begin{array}{l}\text { Pesticides (b) } \\
\left(\mu \mathrm{g} \cdot \mathrm{kg}^{-1}\right)\end{array}$ & 0 & 14 & & & & 0 \\
\hline Indice de pollution & 4 & 3 & 2 & 1 & 1 & 1 \\
\hline
\end{tabular}

(a) Peyrot-Clausade, communication personnelle. (b) Cariès [2]. (c) Fraizier et al. [8]. (d) Ricard [28]. (e) Charpy et al. [4]. (f) CharpyRoubaud et al. [5]. (g) Gabrié et Salvat [9]. 


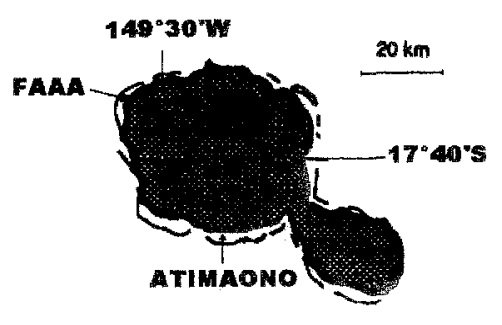

TAHITI

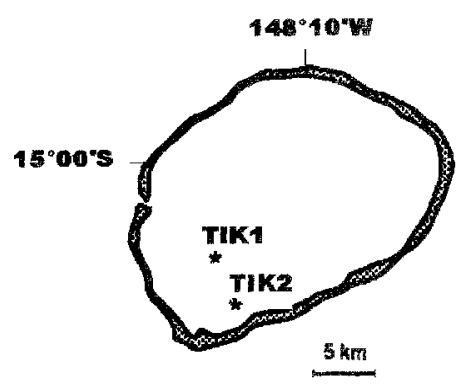

TIKEHAU
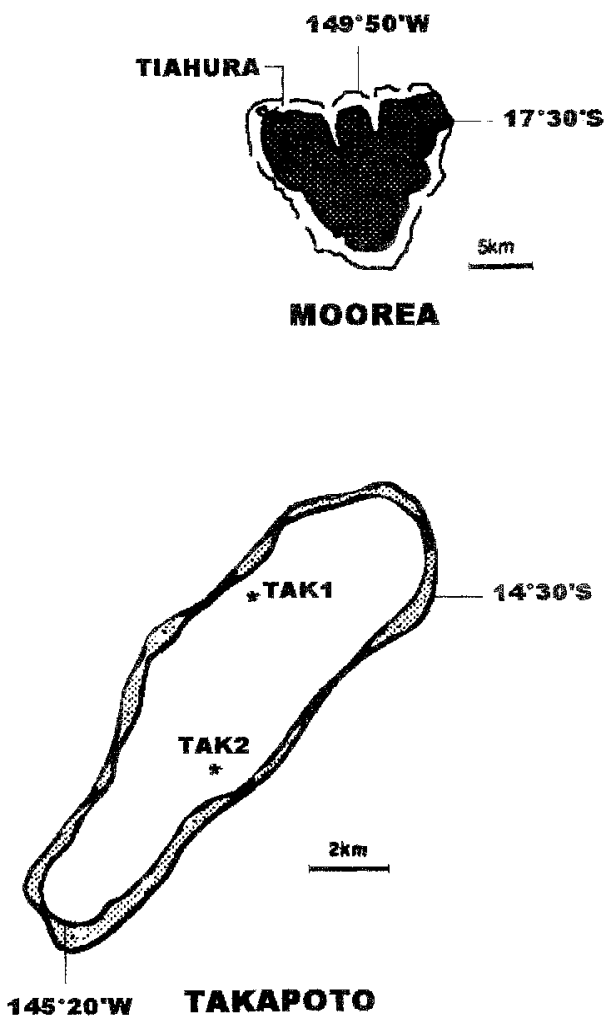

monté sur lame (huit pour chaque bloc, rrois pour les substrats naturels), puis observé au microscope photonique afin d'identifier les cyanobactéries et les différents groupes qui composent le feutrage algal épilithe et endolithe.

La méthode du comptage par point définie par Webel et al. [35] n'a pu être utilisée sur nos échantillons, où après cinq ans de colonisation, la flore endolithe et épilithe constitue un maillage dense inextricable. Une autre technique, probablement plus réaliste dans ce cas, a été adoptée. La lame est observée attentivement. Les taxons sont identifiés et leur importance relative estimée sur l'ensemble de la lame. On obtient ainsi la part que représente chaque taxon de la communauté algalc obscrvéc, exprimée en pourcentage de recouvrement. Ces taxons sont classés suivant les différents groupes: Chlorophycées, Phéophycées, Rhodophycées et cyanobactéries.

\subsubsection{Bioérosion}

La bioérosion des blocs expérimentaux est calculée par différence entre leur volume initial, mesuré lors de leur 
fixation dans les stations, et le volume final, mesuré cinq ans plus tard, lors de leur prélèvement. Cette différence de volume est exprimée en pourcentage de perte de $\mathrm{CaCO}_{3}$.

\subsection{Analyses statistiques des données}

I.e nombre réduit d'échantillons par station et la forte variabilité des mesures nous ont amenés à utiliser la statistique non paramétrique: test de Kruskal-Wallis (test $\mathrm{H}$ ) et test a posteriori de Mann-Whitney (test U). Une classification des différentes stations a été réalisée en utilisant une analyse hiérarchique ascendante: agrégation suivant la distance moyenne sur matrice de distance euclidienne avec le logiciel Stat ITCF [31].

\section{RÉSULTATS}

\subsection{Biomasse du feutrage algal}

Les résultats des analyses en HPLC de la composition pigmentaire des peuplements épilithes et endolithes des blocs et des substrats naturels sont présentés dans le tableau II. La chlorophylle $a$ est naturellement le pigment dominant du cortège pigmentaire mais sa teneur relative par rapport aux pigments accessoires (chlorophylle $b$ et $c$ ) varie suivant les échantillons. La chlorophylle $b$ est le pigment accessoire le plus abondant (26 à $50 \%$ ), puis vient la chlorophylle $c$ (2 à $11 \%$ ). Les concentrations en pigments dégradés (phéophytines et phéophorbides) ne dépassent pas $5 \%$ du total des pigments.

Pour les substrats expérimentaux, les stations de Tik1 et Tik2, avec 55,97 $\pm 15,05$ et $23,29 \pm 4,58 \mu \mathrm{g} \mathrm{chl} a \mathrm{~cm}^{-2}$, ont une biomasse significativement plus élevée $(\mathrm{U}<1$; $p<0,05$ ), alors que les autres stations ne présentent pas de différences significatives $\left(10,37 \pm 1,34 \mu \mathrm{g} \mathrm{chl} a \mathrm{~cm}^{-2}\right.$ pour Tak1 à 14,91 $\pm 2,93 \mu \mathrm{g} \mathrm{chl} a \mathrm{~cm}^{-2}$ pour Moor).

Dans le milieu naturel, on ne constate pas de différence significative de la biomasse $(\mathrm{H}=8,70 ; p>0,2)$ qui varie pourtant de $6,32 \pm 2,16 \mu \mathrm{g} \mathrm{chl} a \mathrm{~cm}^{-2}$ à Faaa à $27,68 \pm$ $3,38 \mu \mathrm{g} \mathrm{chl} a \mathrm{~cm}^{-2}$ à Moor.

A la station Tik1, la biomasse est significativement plus élevée dans le bloc $(\mathrm{U}=0 ; p<0,05)$ que dans le substrat naturel alors qu'il n'y a pas de différence significative de biomasse entre les blocs et le substrat naturel pour les autres stations, même si la biomasse est en générale plus importante dans le substrat naturel.

\subsection{Composition du feutrage algal}

La composition globale du gazon algal (épilithe et endolithe) dans les blocs expérimentaux est résumée dans la figure 2. Les stations des îles hautes (Atim et Moor) sont caraclérisées par un pourcentage de Chlorophycées significativement plus faible que dans les autres stations $(\mathbf{U}=9,5 ; p<0,05)$ mais qui représente une part relativement importante du peuplement (49 et $47 \%$ respectivement). Les Phéophycées et les cyanobactéries

Tableau II. Moyennes \pm écarts types de la biomasse et du pourcentage des différents pigments du feutrage algal (épilithe + endolithe) dans les substrats expérimentaux $(B)$ et les substrats naturels $(\mathrm{N})$.

Table II. Mean \pm standard deviation of biomass and pigment percentages of the epilithic and endolithic algal on experimental substrates (B) and natural substrates $(\mathrm{N})$.

\begin{tabular}{|c|c|c|c|c|c|}
\hline Station & $\begin{array}{c}\text { Biomasse } \\
\left(\mu \mathrm{g} \mathrm{chl} a \mathrm{~cm}^{-2}\right)\end{array}$ & $\begin{array}{c}\text { Chlorophylle } a \\
(\%)\end{array}$ & $\begin{array}{c}\text { Chlorophylle } b \\
(\%)\end{array}$ & $\begin{array}{c}\text { Chlorophylle } c \\
(\%)\end{array}$ & $\begin{array}{c}\text { Pigments dégradés } \\
(\%)\end{array}$ \\
\hline Faaa N & $6,32 \pm 2,16$ & $72 \pm 4$ & $26 \pm 1$ & $2 \pm 1$ & $0 \pm 0$ \\
\hline Atim B & $13,33 \pm 6,39$ & $58 \pm 5$ & $30 \pm 3$ & $7 \pm 1$ & $5 \pm 2$ \\
\hline $\operatorname{Atim} N$ & $18,11 \pm 9,49$ & $62 \pm 4$ & $32 \pm 7$ & $5 \pm 3$ & $1 \pm 0$ \\
\hline Moor B & $14,91 \pm 2,93$ & $57 \pm 11$ & $36 \pm 8$ & $5 \pm 2$ & $2 \pm 1$ \\
\hline Moor N & $27,68 \pm 3,38$ & $55 \pm 8$ & $38 \pm 7$ & $6 \pm 1$ & $1 \pm 1$ \\
\hline Tak1 B & $10,37 \pm 1,34$ & $54 \pm 7$ & $37 \pm 4$ & $7 \pm 3$ & $2 \pm 1$ \\
\hline Tak1 N & $19,40 \pm 1,23$ & $47 \pm 2$ & $42 \pm 2$ & $9 \pm 0$ & $2 \pm 0$ \\
\hline Tak2 B & $11,94 \pm 1,82$ & $69 \pm 7$ & $26 \pm 5$ & $4 \pm 1$ & $1 \pm 0$ \\
\hline Tak2 N & 21,98 & 58 & 29 & 11 & 2 \\
\hline Tik1 B & $55,97 \pm 15,05$ & $38 \pm 3$ & $50 \pm 6$ & $11 \pm 1$ & $1 \pm 0$ \\
\hline Tik1 N & $26,33 \pm 5,50$ & $45 \pm 1$ & $45 \pm 3$ & $9 \pm 0$ & $1 \pm 0$ \\
\hline Tik2 B & $23,29 \pm 4,58$ & $51 \pm 3$ & $35 \pm 3$ & $13 \pm 1$ & $1 \pm 0$ \\
\hline Tik2 N & $18,00 \pm 2,99$ & $47 \pm 6$ & $42 \pm 11$ & $10 \pm 3$ & $1 \pm 0$ \\
\hline
\end{tabular}


$B$

ATIMAONO

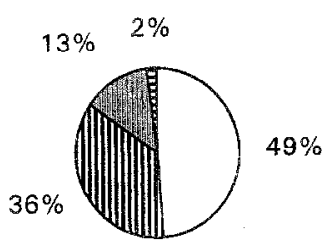

TAKAPOTO 1

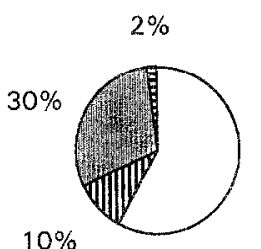

TIKEHAU 1

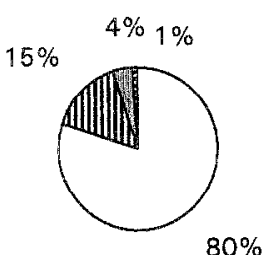

N

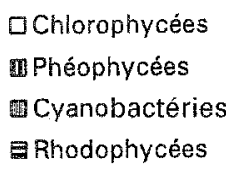

TAKAPOTO 1

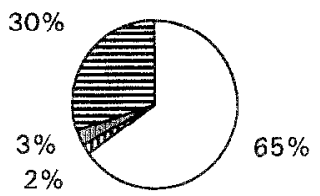

TIKEHAU 1

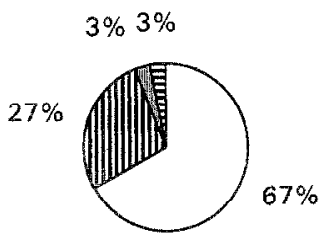

B

MOOREA

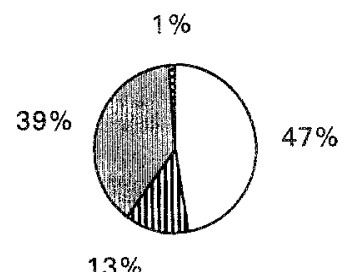

TAKAPOTO 2

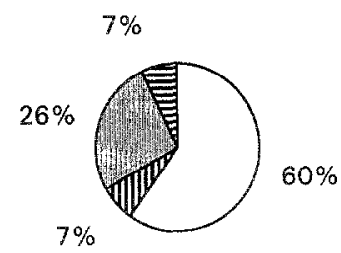

TIKEHAU 2

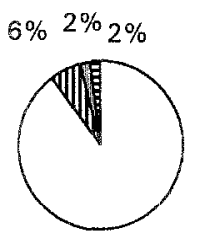

$90 \%$
$N$

MOOREA

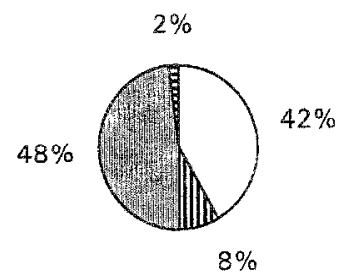

TAKAPOTO 2

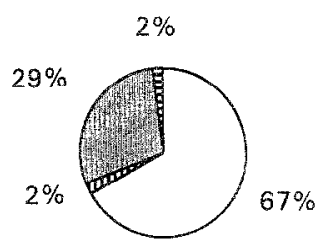

TIKEHAU 2

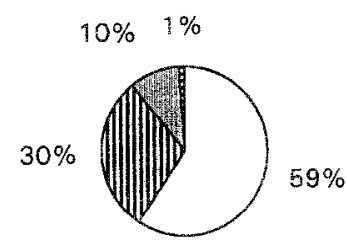

Figure 2. Composition du feutrage algal épilithe et endolithe dans les substrats expérimentaux (B) et naturels (N).

Figure 2. Epilithic and endolithic algal composition on experimental $(B)$ and natural $(\mathrm{N})$ substrates.

représentent 36 et $13 \%$ pour Atim, alors que ces proportions sont inversées pour Moor (13 et $39 \%$ ). Dans ces stations, les espèces endolithes sont moins bicn représcntées qu'aux autres stations, avec seulement 34 à $59 \%$ du peuplement (figure 3). Les stations de Tikehau présentent un pourcentage de Chlorophycées significativement plus élevé $(\mathrm{U}=9 ; p<0,05)$ que dans les autres stations, avec respectivement 80 et $90 \%$ pour Tik1 et Tik2. Par conséquent, les autres groupes sont moins bien représentés, et notamment les cyanobactéries qui sont significativement les plus faibles $(\mathrm{U}=7,5 ; p<0,01)$ et représentent moins de $4 \%$. On constate que dans ces stations de Tikehau l'Ostreobium, seule espèce endolithe, représente une part très importante de l'ensemble de feutrage algal épilithe et endolithe ( 78 et $85 \%$ ) (figure 3). Cette dominance de l'Ostreobium endolithe se retrouve dans les échantillons d'un autre atoll, celui de Takapoto. Les Chlorophycées dominent encore largement avec 58 à $60 \%$ alors que les Phéophycées ( 7 à $10 \%$ ) et les cyanobactéries (26 à $30 \%$ ) sont minoritaires (figure 2). La part de la strate endolithe reste consćquente, avec 66 ct $74 \%$ du feutrage algal (figure 3).

La composition des peuplements algaux colonisant les substrats naturels prélevés à proximité des blocs est présentée également dans le figure 2. Pour les stations des îles hautes, le seul échantillon dont nous disposions, prélevé à Moorea, est très différent de ceux des atolls, sur le plan de la composition floristique. Le pourcentage de cyanobactéries ( $48 \%$ ) est le plus élevé de toutes les stations étudiées. Les Chlorophycées ne représentent que $42 \%$ contre 59 à $67 \%$ dans les autres stations d'atoll. Pour ces dernières, il existe une différence en ce qui concerne le groupe algal le mieux représenté après les Chlorophycées. Il s'agit de $30 \%$ de Rhodophycées à Tak1, mais de $29 \%$ de cyanobactéries pour Tak2 et de 27 ef 


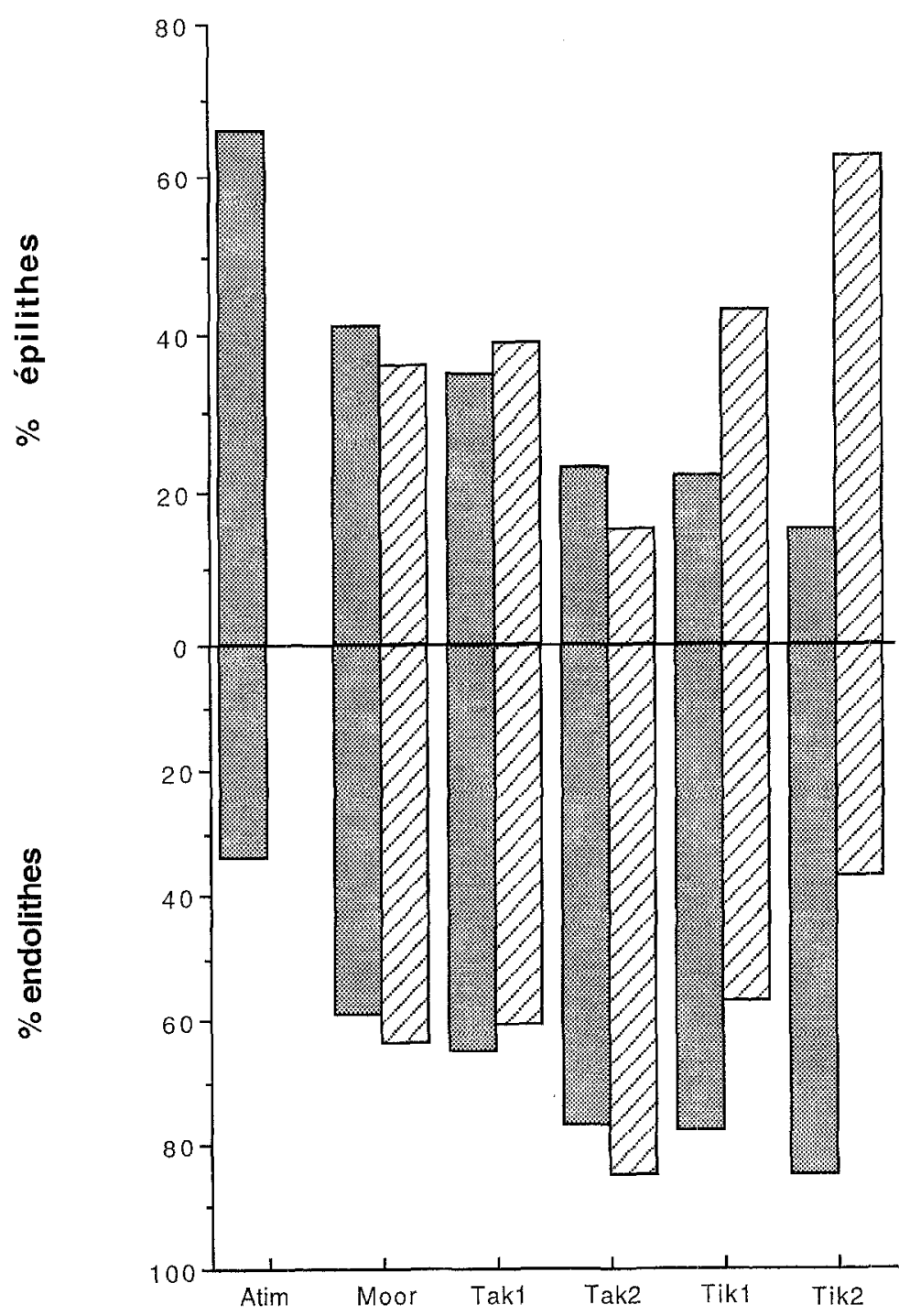

Substrats expérimentaux

Q Substrats naturels

Figure 3. Pourcentage d'épilithes et d'endolithes des substrats expérimentaux et naturels.

Figure 3. Epilithic and endolithic algal percentage on natural and experimental substrates.

$30 \%$ de Phéophycées pour Tik1 et Tik2. Alors que le maximum d'endolithes se trouve à Tak2 $(83 \%)$, il n'est que de 57 à $60 \%$ à Tikl et Tak1 et il est le plus faible à Tik2 (37\%) (figure 3). Cependant, malgré cette variabilité, l'analyse statistique des données ne montre pas de différence significative entre les stations en ce qui concerne la composition floristique des substrats naturels $(\mathrm{H}=2,67 ; p>0,05)$.

Les comparaisons entre substrat naturel et blocs expérimentaux, station par station, nous montrent qu'il n'y a pas de différence significative pour trois d'entre elles: 
Moor $(\mathrm{U}>2 ; p>0,41), \operatorname{Tak} 2(\mathrm{U}>12,5 ; p>0,44)$ et Tik1( $\mathrm{U}>8 ; p>0,24)$. Dans quelques cas, on peut mettre en évidence des différences significatives mais celles-ci portent sur une fraction des peuplements. Ainsi à Tak1, il y a significativement moins de Rhodophycées dans le bloc que dans le substrat naturel ( $\mathrm{U}=1,5 ; p<0,05)$, mais plus de Phéophycées ( $\mathrm{U}=1 ; p<0,05$ ). Pour la station Tik2, il y a significativement plus de Chlorophycées $(\mathrm{U}=0 ; p=0,012)$ et d'endolithes ( $\mathrm{U}=0 ; p=0,012)$ dans le bloc que dans le substrat naturel.

Une classification hiérarchique ascendante basée sur la biomasse végétale, la composition qualitative du peuplement algal et le rapport entre épilithes et endolithes dans les blocs, a permis de regrouper les stations (figure 4 ). Il apparaît un groupe formé des stations de l'atoll de Tike-

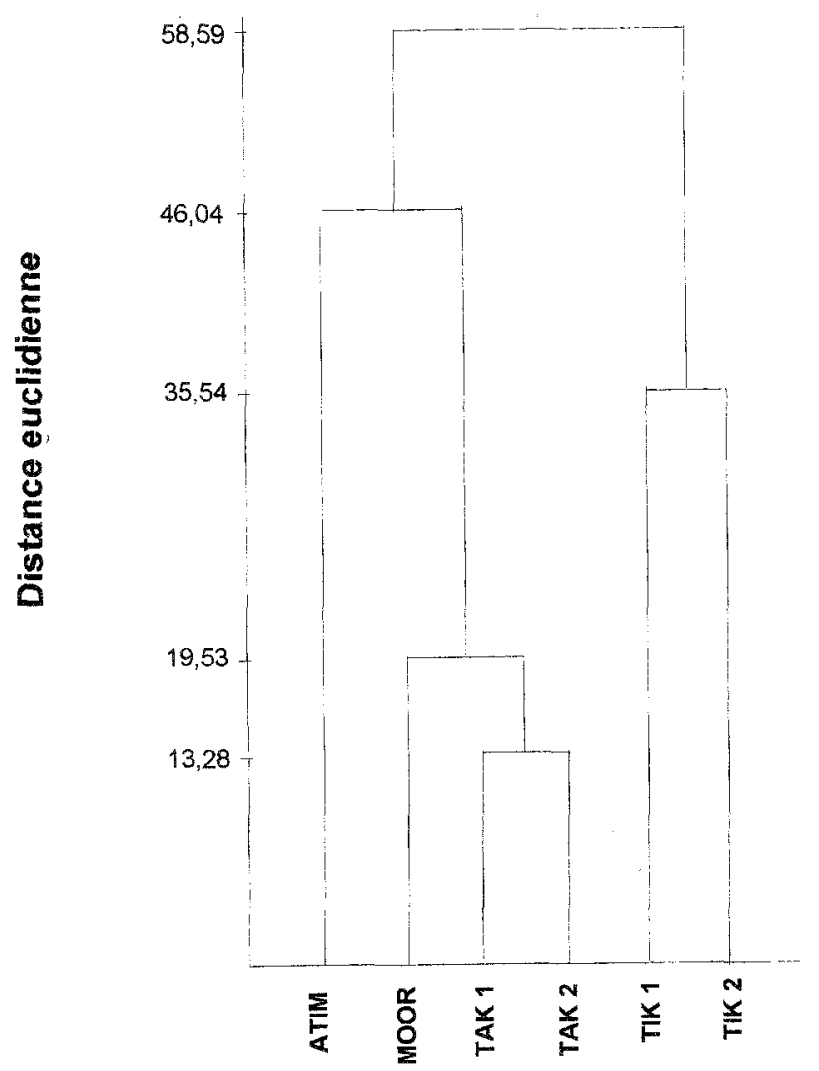

Figure 4. Classification hiérarchique ascendante. Les variables sont la biomasse, les pourcentages de Chlorophycées, de cyanobactéries, de Phéophycées, de Rhodophycées et d'endolithes dans les blocs expérimentaux.

Figure 4. Cluster analysis based on algal biomass, Chlorophyceae, cyanobacteria, Phaeophyceae, Rhodophyceae and endolithic algal percentages on experimental blocks. hau (Tik 1 et Tik2) qui se détache du groupe fomé par l̀es autres stations (distance: 58,59). Les différences entre ces groupes sont dues aux Chlorophycées (35\%), à la biomasse $(26 \%)$, aux endolithes (19\%) et aux cyanobactéries $(18 \%)$. Les deux stations de l'atoll de Tikehau présentent des similitudes entre elles $(d=35,54)$ mais la principale différence entre Tik1 et Tik2 est due, pour $85 \%$, à leur biomasse estimée par la concentration en chlorophylle $a$. Le deuxième groupe, constitué des autres stations, peut lui aussi se diviser en deux sous-groupes. Le premier est la station d'Atimaono et le deuxième est formé de Moor, Tak1 et Tak2. La distance entre ces deux sous-groupes est de 46,04 et les différences sont expliquées à $51 \%$ par les endolithes, $32 \%$ par les Phéophycées et $13 \%$ par les cyanobactéries. Enfin, parmi les dernières stations, Moor est légèrement différente de celles de Takapoto $(\mathrm{d}=19,53)$. Les endolithes, avec $34 \%$, expliquent la plus grande différence, suivi par les cyanobactéries (31\%) et les Chlorophycées (28\%). Parmi toutes les stations, celles de l'atoll de Takapoto (Takl et Tak2) présentent le plus de similitudes (distance $=13,28$ ). La faible différence entre ces stations est expliquée à $36 \%$ par les endolithes, à $28 \%$ par les Phéophycées et $20 \%$ par les Rhodophycées.

\subsection{Relation entre indice de pollution, abondance des espèces endolithes et bioérosion}

A partir des données du tableau I, nous avons défini un indice de pollution de 1 à 4, 1 élant attribué à des eaux «saines» et 4 étant le signe d'une forte pollution. A la station de Faaa, l'indice de pollution, avec une valeur de 4, est maximal. Cette station est située dans le port de Papeete qui reçoit les eaux usées non traitées de cette ville de 80000 habitants. Les concentrations en détergents, en hydrocarbures et en métaux lourds sont cinq fois plus importantes que dans l'océan, et l'on observe jusqu'à 10000 coliformes fécaux par $100 \mathrm{~mL}$ (tableau ). De plus, la station de Faaa se trouve à proximité de l'embouchure de la rivière de Tipearui, qui traverse une zone où sont concentrées de nombreuses usines chimiques polluantes.

La station d'Atimaono est située à plus de $40 \mathrm{~km}$ du port de Papeete, dans une zone peu urbanisée à fort taux de sédimentation. La pollution y est faible par rapport à Faaa, mais les signes d'une dégradation sont nets. Même si l'on ne retrouve pas de pollution bactériologique, les pesticides sont présents dans le sédiment $\left(14 \mu \mathrm{g} \mathrm{kg}^{-1}\right)$, ainsi que les détergents anioniques (10-20 $\left.\mathrm{\mu g} \mathrm{kg}^{-1}\right)$ 
(tableau I). A cette station, l'indice de pollution est de 3, les apports d'alluvions, la turbidité et la sédimentation y sont très importants mais l'hydrodynamisme est faible.

L'île de Moorea est faiblement peuplée (moins de 70 habitants $\mathrm{km}^{-2}$ ). La station Moor, située sur le récif frangeant a un indice de pollution de 2. L'urbanisation et les rejets d'égout en mer sont faibles et il n'y a pas d'industrie chimique polluante.

L'indice de pollution le plus faible (1) est rencontré dans les stations des lagons d'atolls (Takapoto et Tikehau). On ne peut pas parler de pollution ou d'eutrophisation pour ces stations qui sont alors considérées comme caractéristiques d'eaux « saines».

Si l'on représente le pourcentage d'espèces endolithes dans le feutrage algal en fonction des stations et de l'indice de pollution (figure 5), on constate que le pourcentage d'endolithes augmente des îles hautes vers les atolls en même temps que la qualité des eaux s'améliore. Ce pourcentage cst minimal à Atim (34\%), qui présente un indice de pollution de 3 , alors que le maximum est rencontré dans les atolls et notamment à Tik2 $(85 \%)$ où l'indice de pollution n'est que de 1.

Une estimation de la bioérosion globale des blocs expérimentaux a été réalisée sur la base des pertes de $\mathrm{CaCO}_{3}$ en cinq ans. Ces pertes sont les plus importantes à Faaa où le bloc a complètement disparu en moins de cinq ans. La bioérosion est importante également à Tikehau (67 et $80 \%$ de perte). Dans les deux autres stations des îles hautes, Atim et Moor, les pertes sont de 30 et $35 \%$ et elles sont les plus faibles pour l'atoll de Takapoto (12 et $22 \%$ ). On a tenté de représenter la distribution des différentes stations étudiées en fonction de l'importance de la bioérosion et de l'indice de pollution que nous avons défini (figure 6). La bioérosion est maximale à Faaa, qui est la station la plus polluée, avec le plus d'organismes brouteurs; elle diminue dans les autres stations des îles hautes (Atim et Moor), parallèlement à la pollution, pour être minimale dans l'atoll de Takapoto. Les stations de l'atoll de Tikehau se détachent de ce schéma par leur pourcentagc de bioćrosion. Pour ccs stations, la présence de très grandes quantités d'éponges perforantes dans les blocs

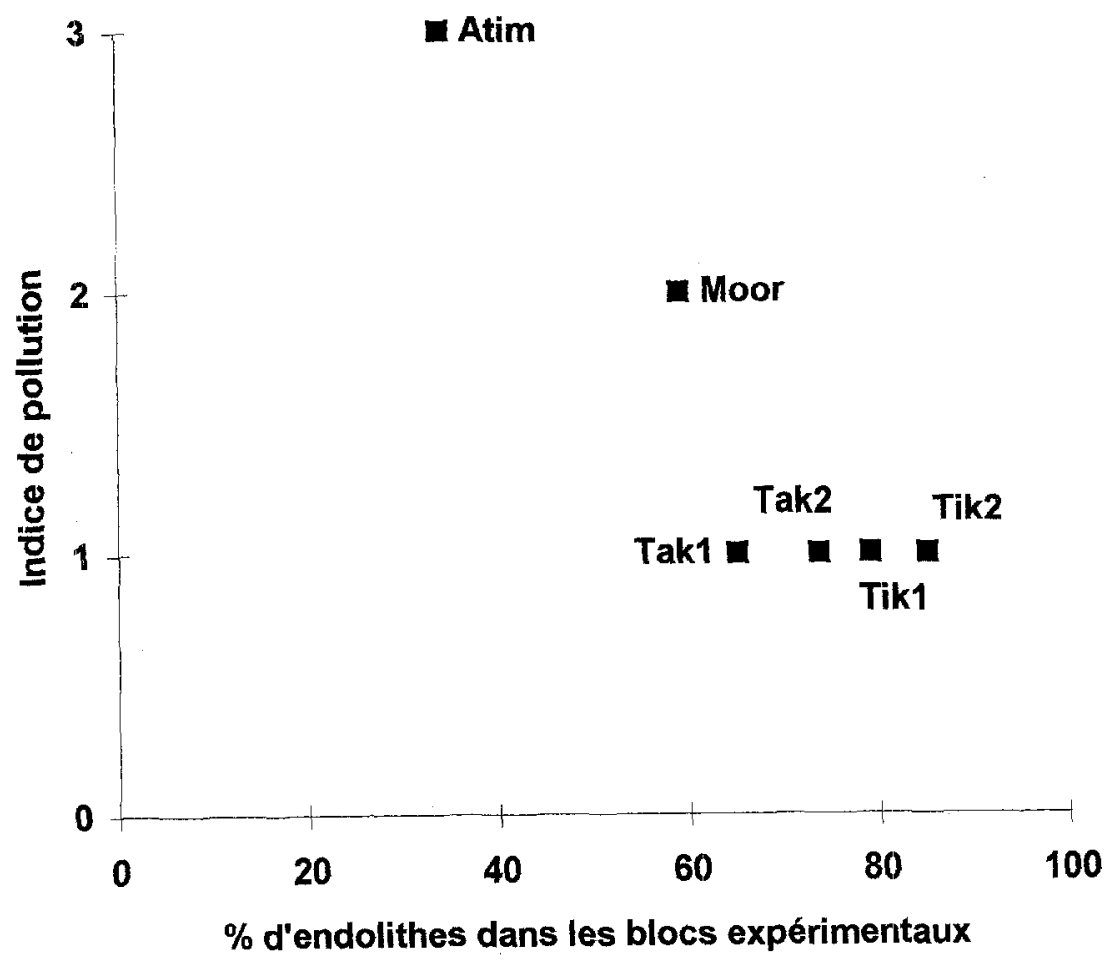

Figure 5. Répartition des stations d'études en fonction du pourcentage d'endolithes dans les blocs expérimentaux et d'un indice de pollution.

Figure 5. Distribution of the study sites according to the percentage of endolithic algae in the experimental blocks and a pollution index. 


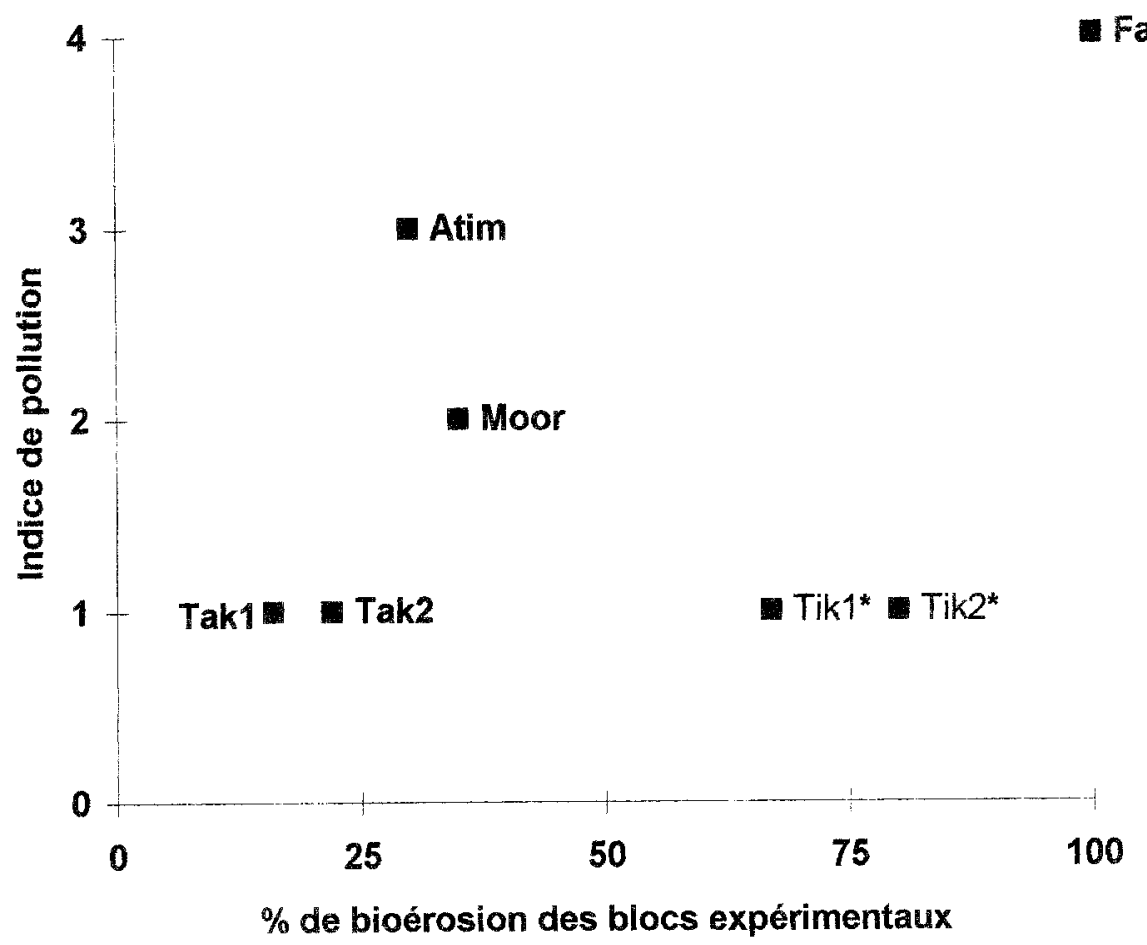

Figure 6. Répartition des stations d'études en fonction du pourcentage de bioérosion des blocs expérimentaux et d'un indice de pollutiont. Blocs soumis à une très forte bioérosion interne due aux éponges purifiantes.

Figure 6. Distribution of the study sites according to the percentage of bioerosion in the experimental blocks and a pollution index. Blocks with maximum internal bioerosion by sponges.

entraîne une bioérosion très forte qui, dans ce cas, est presque exclusivement interne, alors que dans les autres stations la bioérosion est principalement externe, due aux oursins et aux Scaridés.

\section{DISCUSSION}

I es résultats concernant la composition et la biomasse du feutrage algal épilithe et endolithe permettent de différencier nettement les stations des îles hautes et des atolls. On constate une diminution de la biomasse microphytique totale (épilithes + endolithes) des îles hautes vers les atolls. Ce résultat peut s'expliquer par l'enrichissement des eaux des îles hautes en nutriments (tableau I) d'origine terrigène et anthropique qui permet un développe ment important de la biomasse algale microphytique. Dans ce cas, le feutrage algal est principalement constitué d'espèces épilithes, alors que la contribution des espèces endolithes est modeste. Mais, bien que la strate épilithe soit dominante, la hauteur de ce gazon végétal reste faible car la pression due aux importants effectifs des brouteurs est forte [27]. Dans ces conditions, on peut penser que les épilithes n'ont pas le temps de se développer en hauteur avant d'être broutés. Des résultats similaires ont été mis en évidence dans la Grande Barrière de corail $[15,30]$ et dans les Caraïbes [10,34]. Il s'agit ici des premières observations en Polynésie française dans ce domaine. Au contraire, les deux stations de l'atoll de Takapoto, où les eaux du lagon montrent un caractère oligotrophe accentué [32], présentent le minimum de biomasse constituée essentiellement par des espèces endolithes. Ceci peut s'expliquer par le fait que c'est un atoll fermé où le temps de résidence de l'eau est de six ans. La quantité de sels nutritifs dans les eaux n'est pas le seul facteur contrôlant le développement du feutrage algal épilithe et endolithe. En effet, dans les stations d'un autre atoll (Tikehau) on trouve la plus forte biomasse, notamment à la station

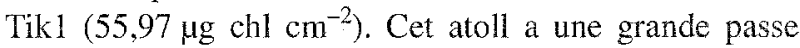
permettant des apports permanents en nutriments dans le lagon. Le déficit en nutrilites constaté dans le lagon (tableau I) pourrait être dû à une consommation par la biomasse végétale, le renouvellement de l'eau ne compensant que partiellement cette consommation. 
En ce qui concerne la bioérosion, les résultats montrent que non seulement le feutrage algal épilithe et endolithe participe directement à ce mécanisme, mais que la présence d'un feutrage algal épilithe important favorise l'implantation des macroperforants en piégeant leurs larves [27]. Lors de leur développement, ces larves contribuent à accentuer la bioérosion, comme sur les blocs expérimentaux de cinq ans où la plus grande quantité de macroperforants est observée dans les stations où la couverture épilithe est la plus développée. Inversement, dans ces stations, les microperforants végétaux sont plus faiblement représentés. Il semble donc exister une relation inverse entre la richesse des peuplements endolithes et celle de la strate épilithe. L'augmentation parallèle des brouteurs (oursins) et des épilithes s'accompagne d'une diminution de la présence des microperforants dans des milieux enrichis en particules et nutrilites.

Nos résultats amènent à penser que l'importance quantitative du feutrage algal, ainsi que l'importance relative de la strate épilithe et endolithe, dépendent de la synergie de nombreux facteurs écologiques du milieu. Ce sont notamment la densité des brouteurs, les ressources trophiques pour la biomassc végćtalc (nutrilites), le degré d'anthropisation des lieux d'implantation des blocs, et au final, la pollution des eaux. Dans l'île de Tahiti, à la station de Faaa correspondant au port de Papeete, l'indice de pollution des eaux est maximal (4). On sait que des indices de forte pollution s'accompagnent généralement d'un enrichissement en azote, lequel peut permettre le développement d'un feutrage algal épilithe très dense [24]. Cela se traduit par une multiplication des organismes se nourrissant de cette strate, notamment des oursins brouteurs tel que Echinometra mathaei $\left(44 \pm 14\right.$ ind $\mathrm{m}^{-2} \mathrm{Pey}-$ rot-Clausade, comm. pers.). La bioérosion qui en résulte est très intense si bien que les blocs, à cette station, ont complètement disparu en moins de cinq ans.

A la seconde station de l'île de Tahiti (Atim), l'indice de pollution est encore important (3). Le bloc est caractérisé par la plus grande abondance relative d'épilithes rencontrés dans cette étude ( $64 \%$ ). La forte sédimentation et la turbidité des eaux ne permettent peut-être pas à la strate endolithe de recevoir assez de lumière pour un développement optimal. La présence d'Echinometra, si elle est faible, n'est pas négligeable $\left(<1\right.$ ind $\mathrm{m}^{-2}$, PeyrotClausade, comm. pers.) et semble attestée par la présence des formes dégradées de pigments chlorophylliens (phéophytines et phéophorbides). En effet, ces produits sont le résultat de la digestion acide du feutrage algal et se rencontrent notamment en grandes quantités dans les fèces des oursins, ainsi que dans leurs tractus digestifs [13].

L'île de Moorea, avec un indice de pollution de 2, présente beaucoup de similitudes avec celle d'Atimaono en ce qui concerne la concentration en pigments, mais en diffère par un rapport épilithes/endolithes inversé, soit $59 \%$ d'endolithes (contre $34 \%$ à Atim). Les eaux étant claires, le développement des endolithes est facilité. Cette station a pratiquement les mêmes caractéristiques que celle de l'atoll de Takapoto, mais elle est plus riche en épilithes et sa concentration en pigments est plus importante. Il y a un enrichissement des eaux à Moorea, île haute, par le lessivage des sols, ce qui expliquerait une plus forte production.

Pour les atolls de Takapoto et Tikehau, on ne peut pas parler de pollution anthropique [27,32] et l'indice de pollution est minimal (1). Dans ce cas, les ressources en sels nutritifs paraissent insuffisantes pour entretenir un important feutrage algal épilithe, ce qui pourrait expliquer que dans ces lagons il n'y a pas d'oursin et que les brouteurs ne sont constitués que de quelques Scaridés [26]. La biomasse végétale est donc constituée essentiellement d'endolithes.

On peut penser que lintensité de la bioérosion externe, liée au gradient de pollution, influence la proportion d'endolithes dans le feutrage algal. En présence d'une bioérosion externe importante par les organismes brouteurs, une partie de la strate endolithe est consommée. Les mesures effectuées correspondent alors à la strate endolithe "résiduelle". On sous-estime donc la contribution des espèces endolithes au feutrage algal. Rose et Risk [28] ont démontré une corrélation entre la pollution organique et l'augmentation de la bioérosion. L'eutrophisation serail une cause de déclin du réci par l'augnnentation de la bioérosion [11]. Elle stimulerait la production primaire mais limiterait la calcification des récifs [13]. L'eutrophisation entraîne une augmentation de la production du feutrage permettant, à son tour, une augmentation du nombre des brouteurs. Rose et Risk [29] montrent, dans ces conditions de milieux, une augmentation de la quantité de coraux morts et du taux de recouvrement du gazon algal épilithe, qui se développe uniquement sur ces substrats morts. Les brouteurs, disposant alors de plus de nourriture, se multiplient et leurs effets bioérodeurs s'amplifient. Il y a donc un effet de synergie entre l'eutrophisation, le développement du feutrage algal épilithe et des brouteurs, et la bioérosion. A La Réunion, il a été observé que la bioérosion était plus forte dans les stations où le feutrage algal était le plus développé et le nombre 
d'oursin le plus élevé, mais dans ce cas, les stations correspondaient aux sites les moins eutrophes [6, 7]. Une augmentation de l'eutrophisation des eaux se traduisait alors par un développement d'algues calcaires encroûtantes et de Lobophora (Phéophycées) qui ne sont pas attractives pour les oursins, ce qui explique leur faible nombre dans ces stations et donc la faible bioérosion.

La part que représentent les endolithes dans le feutrage algal augmente des îles hautes vers les atolls. Dans les stations des îles hautes, les endolithes comptent pour moins de $60 \%$. Ces endolithes sont constitués de cyanobactéries et de Chlorophycées. Le Mastigocoleus (cyanobactérie) n'est présent que dans ces stations où le broutage est important. Or c'est une espèce pionnière [19], parmi les premières qui se développent sur la surface des blocs continuellement remaniée dans ces stations. Les cyanobactéries, souvent constituées d'espèces pionnières, sont abondantes dans les stations des îles hautes, où la bioérosion externe est forte, alors que dans les stations où la bioérosion externe est faible, elles sont remplacées par la Chlorophycées Ostreobium qui devient majoritaire dès la deuxième année de colonisation [19]. Cette tendance se confirme dans les blocs étudiés ici, âgés de cinq ans, et où l'Ostreobium représente de 59 à $100 \%$ des endolithes. A Takapoto, la flore endolithe, constituée de cyanobactéries et d'Ostreobium, représente $70 \%$ de l'ensemble du feutrage algal. A Tikehau, les endolithes représentent plus de $78 \%$ du peuplement et sont constituées uniquement d'Ostreobium.

Très peu d'études ont été réalisées sur la contribution des espèces endolithes à la production primaire récifale, du fait de la difficulté des observations. Certains auteurs ont même émis l'hypothèse que, par manque de lumière à l'intéricur du substrat, la production des endolithes était négligeable $[11,33]$. Mais les microperforants sont présents dans tous les substrats carbonatés (coraux morts, coquilles, grains de sédiments) et la Chlorophycée Ostreobium colonise même les coraux vivants [18]. Wanders [34] signale l'importance de la contribution des endolithes à la production. Payri [25], à Moorea, a constaté que les endolithes, qui ne représentaient que $0,05 \%$ de la biomasse algale étudiée, participaient à hauteur de $21 \%$ de la production photosynthétique nette journalière. On voit bien l'importance de la strate endolithe dans la production primaire qui représente, dans notre étude, de 34 à $85 \%$ du peuplement algal. Cependant, il est difficile d'établir des comparaisons car les unités et les paramètres pour estimer la biomasse sont rarement les mêmes. A Hawaï, Morrissey [22] indique une production pri- maire du gazon algal de $0,11 \mathrm{~g} \mathrm{~m}^{-2} \mathrm{j}^{-1}$ de carbone. Payri [25] évalue à $4 \mathrm{~g} \mathrm{~m}^{-2} \mathrm{j}^{-1}$ de carbone la production primaire des gazons, alors que dans les récifs de la Grande Barrière de Corail, Klumpp et McKinnon [16] ont mesuré une production primaire du gazon algal variant de 1 à $2,5 \mathrm{~g} \mathrm{~m}^{-2} \mathrm{j}^{-1}$ de carbone.

Il est donc difficile de comparer nos résultats à ceux des autres auteurs, en particulier pour l'estimation de la biomasse algale, par les mesures de pigments en HPLC. Cette approche avait en outre pour but de décrire la relation entre le cortège pigmentaire et le cortège floristique. Mais, pour mesurer avec certitude cette biomasse algale, il paraît indispensable, dans l'avenir, de déterminer une standardisation des méthodes.

La comparaison entre le feutrage algal des blocs et du substrat naturel sur lequel ils sont implantés tend à montrer que la colonisation des blocs est représentative du milieu naturel.

Cependant, des techniques plus précises restent à mettre au point pour quantifier avec exactitude le feutrage algal endolithe et son pourcentage de recouvrement. Le comptage par point sous microscope optique, le plus souvent utilisé, n'est pas approprié après un temps aussi long de colonisation, à cause de la densité et de la complexité des peuplements. La technique de comptage beaucoup plus rudimentaire que nous avons appliquée est peut être mieux adaptée à ces conđitions.

\section{CONCLUSION}

De cette étude il ressort une grande variabilité dans la composition et la biomasse des deux strates des peuplements constituant le feutrage algal dans les stations de Polynésie française. Deux facteurs conditionnent le développement de ces peuplements : la nature géomorphologique des sites (île haute ou atoll) et la bioérosion, liée au degré de pollution des eaux, à l'eutrophisation el à l'intensité du broutage.

Les stations des îles hautes sont caractérisées par un degré d'eutrophisation et un nombre d'organismes brouteurs importants. Cela se traduit par une augmentation de la biomasse du feutrage algal et de la contribution des épilithes dans ce feutrage. Au contraire, les stations des lagons d'atoll, souvent très oligotrophes, ne permettent probablement pas le développement d'un feutrage algal important dont la biomasse est plus faible que dans les îles hautes. Les endolithes sont majoritaires dans ce peu- 
plement algal et constituées principalement de la Chlorophycées Ostreobium. La pauvreté en épilithes paraît expliquer l'absence des oursins dans ces lagons et la très faible bioérosion.

Dans la succession écologique, les premiers microperforants qui s'installent sont les cyanobactéries, espèces pionnières, qui sont peu à peu remplacées par la Chlorophycée Ostreobium. Cette algue demande moins de lumière et, pour s'installer, profite peut être de l'augmentation de la porosité du substrat due aux cyanobactéries. Une fois qu'elle est en place, la compétition trophique bascule en faveur de l'Ostreobium.

La participation de la strate endolithe (dont le rôle avait été négligé auparavant) est mise en évidence dans la biomasse algale (de 34 à $85 \%$ ). Les auteurs proposent d'utiliser le feutrage algal comme indicateur de la qualité et de la santé d'un récif. Ils ont montré que plus les eaux sont saines et oligotrophes, plus la strate endolithe est importante et monospécifique. En revanche, quand les eaux sont eutrophes, la bioérosion externe et la biomasse algale sont plus fortes, la strate épilithe devient majoritaire et diversifiée alors que la strate endolithe "résiduelle" diminue.

\section{Remerciements}

Ce travail a été réalisé dans le cadre d'un programme de coopération franco-australienne "Sciences marines" (responsable, M. Peyrot-Clausade) et DITAC (responsable, P. Hutchings).
[1] Bruggemann J.H., Kessel A.M.V., Breeman A.M., Bioerosion by parrotfishes: implications of feeding mode, food preference and skeletal density of grazing substrates for the generation and reworking of sediments, in: First European Regional Meeting, Int. Soc. Reef Studies, Vienna, Austria (1993) 11 p.

[2] Cariès J.C., Surveillance de la qualité du milieu marin dans la circonscription du Port autonome de Papeete et autour de Tahiti dans le cadre du Réseau territorial d'observation, Proc. ISRS Nouméa (1991) 17-24.

[3] Carpenter R.C., Partitioning herbivory and its effects on coral reef algal communities, Ecol. Monogr. 56 (1986) 345-363.

[4] Charpy L., Phytoplankton biomass and production in two Tuamotu atoll lagoons (French Polynesia), Mar. Ecol. Prog. Ser. 145 (1996) 133-1.42.

[5] Charpy-Roubaud C.J., Charpy L., Cremoux J.L., Nutrient budget of the lagoonal waters in an open central South Pacific atoll (Tikehau, Tuamotu, French Polynesia), Mar. Biol. 107 (1990) 63-73.

[6] Chazottes V., Étude expérimentale de la bioérosion et de la sédimentogénèse en milieu récifal: effets de l'eutrophisation (île de La Réunion, Océan Indien occidental), Thèse de doctorat de l'université de Provence (Aix-Marseille I), 1994, 285 p.

[7] Chazottes V., Étude expérimentale de la bioérosion et de la sédimentogénèse en milieu récifal: effets de l'eutrophisation (île de La Réunion, Océan Indien), C. R. Acad. Sci. 323 (1996) 787-794.

[8] Fraizier A., Franck D., Benente P., Debiard J.P., Observations on the various form of pollution of Tahiti lagoons, Proc. 5th. Int. Coral Reef Congress, Tahiti, 6 (1985) 445-451.
[9] Gabrié C., Salvat B., General features of $\Gamma$ rench Polynesian Isiands and their coral reefs. Proc. 5th Coral, Reef Congr., Tahiti, 1 (1985) 1-16.

[10] Hackney J.M., Carpenter R.C., Adey W.H., Characteristic adaptations to grazing among algal turfs on a caribean coral reef, Phycologia 28 (1989) 109-119.

[11] Halldal P., Photosynthetic capacities and photosynthetic action spectra of endozoic algae of the massive coral Favia, Biol. Bull. 134 (1968) 411-424.

[12] Hallock P., The role of nutrient availability in bioerosion: consequences to carborate buildups, Palaeogeogr. Palaeoclim. Palaeoecol. 63 (1988) 275-291.

[13] Harmelin-Vivien M., Peyrot-Clausade M., Romano J.C., Transformation of algal turf by echinoids and scarid fishes on French Polynesia coral reefs, Coral Reefs 11 (1992) 45-50.

[14] Kinsey D.W., Coral reef system response to some natural and anthropogenic stresses, Galaxea 7 (1988) 113-128.

[15] Klumpp D.W., Polunin N.V.C., Algal production, grazers and habitat partitioning on a coral reef: positive correlation between grazing rate and food availability, Proc. 24th Europ. Mar. Biol. Symp. (1990) 372-388.

[16] Klumpp D.W., McKinnon A.D., Temporal and spatial patterns in primary profuction of a coral-reef epilithic algal community, J. Exp. Mar. Biol. Ecol. 131 (1989) 1-22.

[17] La Giraudière I., Laborbe P., Romano J.C., HPLC determination of chlorophylls and breackdown-products in surface microlayers, Mar. Chem. 26, (1989) 189-204.

[18] Le Campion-Alsumard T., Campbell S.E., Golubic S., Endoliths and the depth of the photic zone-discussion, J. Sedim. Petrol. 52 (1982) 1333-1334. 
[19] Le Canpion-Alsunard T., Golubic S., Hutclings P., Microbial endoliths in skeletons of live and dead corals: Porites lobata (Moorea, French Polynesia), Mar. Ecol. Prog. Ser. 117 (1995) $149-157$.

[20] Le Campion-Alsumard T., Romano J.C., Peyrot-Clausade M., Le Campion J., Paul R., Influence on some coral recf communities on the calcium carbonate budget of Tiahura reef (Moorea, French Polynesia), Mar. Biol. 115 (1993) 685-693.

[21] Mantoura R.F.C., Llewellyn C.A., The rapid determination of algal chlorophyll and carotenoid pigments and their breakdown products in natural water by reverse-phase high performance liquid chromatography, Anal. Chim. Acta. 151 (1983) 297-314.

[22] Morrissey J., Primary productivity of coral reef benthic macroalgae, Proc. 5th Int. Coral Reef Cong., Tahiti, 5 (1985) $77-82$.

[23] Morrison D., Comparing fish and urchin grazing in shallow and deeper coral reef algal communities, Ecology 69 (1988) $1367-1382$.

[24] Naim O., Seasonal responses of a fringing reef community to eutrophication (Reunion Island, Western Indian Ocean), Mar. Ecol. Prog. Ser. 99 (1993) 137-151.

[25] Payri C.E., Variabilité spatiale et temporelle de la communauté des macrophytes des récifs coralliens de Moorea (Polynésie française). Contribution des algues au métabolisme du carbone de l'écosystème récifal, Thèse de doctorat de l'université des Sciences et Techniques du Languedoc, 1987, 149 p.

[26] Peyrot-Clausade M., Le Campion-Alsumard T., HarmelinVivien M., Romano J.C., Chazottes V., Pari N., Le Campion J., La bioérosion dans le cycle des carbonates: essais de quantification des processus en Polynésie française, Bull. Soc. Géol. France 1 (1995) 85-94.

[27] Peyrot-Clausade M., Le Campion-Alsumard T., Hutchings P., Le Campion J., Payri C., Fontaine M.F., Initial bioerosion and bivaccretion on experimental substrates in high island and atoll lagoons (French Polynesia), Oceanol. Acta 18 (1995) $531-541$.

[28] Ricard M., Some effect of dredging on the primary production of the Tiahura lagoon in Moorea (Society Island, French Polynesia), Proc. 4th. Int. Coral Reef Symposium, Manila 4 (1981) $431-436$.

[29] Rose C.S., Risk M.J., Increase in Chona delithrix infestation of Montastrea cavernosa heads on a organically polluted portion of the Grand Cayman fringing reef. Mar. Ecol. 6 (1985) 345-363.

[30] Scott F.J., Russ G.R., Effects of grazing on species composition of the epilithic algal community on coral reefs of the central Great Barrier Reef, Mar. Ecol. Prog. Ser. 39 (1987) 293304.

[31] Stat-ITCF Manuel d'utilisation, Institut Technique des Céréales et des Fourrages, Paris (1991) 267p.

[32] Vacelet E., Amoux A., Thomassin B.A., Particulate material as an indicator of pearl-oyster excess in Takapoto lagoon (Tuamotu, French Polynesia), Aquaculture 144 (1996) 133-148.

[33] Wanders J.B.W., The role of benthic algae in the shallow reef of Curaçao (Netherlands Antilles) I: primary productivity in the coral reef, Aquat. Bot. 2 (1976) 235-270.

[34] Wanders J.B.W., The role of benthic algae in the shallow reef of $C^{2}$ uraçao (Netherlands Antilles) III: the significance of grazing, Aquat. Bot. 3 (1977) 357-390.

[35] Webel E.R., Kistle G.S., Scherler W.F., Pratical stereologica] methods for morphometric cytology, J. Cell. Biol. 30 (1966) 23-28.

[36] Williams S.L., Carpenter R.C., Photosynthesis/photon flux density relationships among components of coral reef algal turfs, J. Phycol. 26 (1990) 36-40. 